\title{
Automatic Generation of Robot and Manual Assembly Plans Using Octrees
}

\author{
H. Medellín ${ }^{1}$, J. Corney ${ }^{2}$, J. M. Ritchie ${ }^{3}$, T. Lim ${ }^{3}$ \\ ${ }^{1}$ Facultad de Ingeniería, Universidad Autónoma de San Luis Potosí, 78290, San Luis Potosí, Mexico. \\ ${ }^{2}$ University of Strathclyde, G1 $1 X Q$, Glasgow, Scotland, UK \\ ${ }^{3}$ Mechanical Engineering Department, Heriot-Watt University, EH14 4AS, Edinburgh, Scotland, UK.
}

\begin{abstract}
Purpose - This paper aims to investigate automatic assembly planning for robot and manual assembly.

Design/methodology/approach - The octree decomposition technique is applied to approximate CAD models with an octree representation which are then used to generate robot and manual assembly plans. An assembly planning system able to generate assembly plans was developed to build these prototype models.
\end{abstract}

Findings -Octree decomposition is an effective assembly planning tool Assembly plans can automatically be generated for robot and manual assembly using octree models.

Research limitations/implications - One disadvantage of the octree decomposition technique is that it approximates a part model with cubes instead of using the actual model. This limits its use and applications when complex assemblies must be planned, but in the context of prototyping can allow a rough component to be formed which can later be finished by hand.

Practical implications - Assembly plans can be generated using octree decomposition, however, new algorithms must be developed to overcome its limitations. 
Originality/value - This paper has proved that he octree decomposition technique is an effective assembly planning tool. As a result, an assembly planning system has been developed. Assembly plans for automatic and manual assembly can be generated automatically by the proposed system, which is a novelty since there are no fully automatic assembly planning systems for manual assembly reported in the literature.

Keywords Manual assembly, Robot assembly, Assembly plan, Octree decomposition Paper type Research paper

\section{Introduction}

The use of traditional manufacturing processes such as CNC machining and robotic systems as a way for rapid manufacturing has attracted growing interest in recent years. An integration of industrial robots with RP technologies as a flexible rapid prototyping cell was proposed by Gibson (1996), Hsuan-kuan and Lin Grier (2003), and Chen and Song (2001). The integration of rapid prototyping techniques with CNC machining was presented by Karunakaran et al. (2000), Junghoon et al. (2002), and Frank et al. (2002). Recently, the authors reported the use of Octree approximations as an approach for low cost manufacturing and visualization of CAD models Medellín et. al. (2006, 2008). Their proposed OcBlox technique is based on the Octree approximation and assembly of CAD models with cubes of different sizes and materials. An overview of the OcBlox system is shown in Fig. 1. This paper describes the assembly planning system developed to support the OcBlox system This assembly planning system is able to generate assembly plans for either robot or manual assembly of a component. 


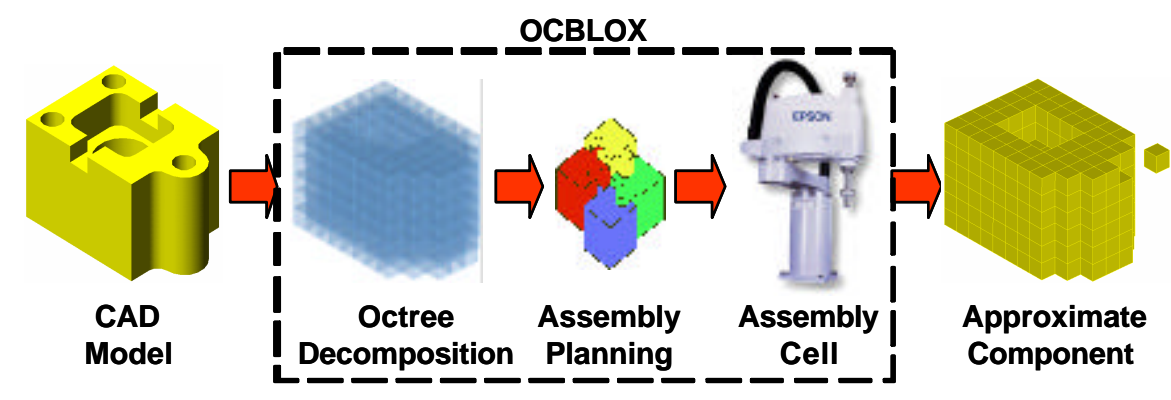

Figure 1. Overview of the OcBlox system.

The rest of this paper is organized as follows. Section 2 provides a literature review regarding the assembly planning and the octree decomposition. Section 3 describes the robotic assembly cell developed to carry out the assemblies. A description of the OcBlox assembly planning system is presented in section 4. The results obtained from the implementation and testing of the system are discussed in section 5. Finally the paper ends with the conclusions drawn from the research work.

\section{Literature review}

Assembly is an important stage in product development and accounts for a large proportion of the manufacturing costs. However, assembly remains one of the least understood manufacturing processes, Whitney et. al. (1999). Most assembly design in industry is based on the mating, aligning or offsetting of the regular faces of each of the mating parts of the assembly, Lin and Farahati (2003). The following paragraphs summarise some research work related to the assembly planning (Homem and Lee, 1991; Kai-I and Tai-His, 1995; Schmidt and Jackman, 1995; Swaminathan and Suzanne, 1996; Kaufman et. al., 1996; Wilson, 1996; Sundaram et. al., 2001; Mascle, 2002).

\subsection{Automatic assembly planning}

According to Homem and Lee (1991), the most important technical issues addressed in automated 
assembly planning are: assembly sequence representation, generation and evaluation; planning process accuracy and efficiency; CAD program integration; and task and motion planner integration. A classification of assembly representation methods reported in the literature is shown in Table 1 . Assembly sequence generation has primarily focused on algorithms for the fast and efficient generation of feasible assembly plans. Most of the assembly sequence generators transform the problem of generating assembly sequences into the problem of generating disassembly sequences. A categorization of the assembly sequence generation methods reported in the literature is shown in Table 2 . Testing the feasibility of assembly plans must consider several feasibility issues such as: geometrical, mechanical, manipulability, accessibility, stability, visibility, and material. Geometrical feasibility is one of the most important constraints because it checks if the assembly or removal of a part will collide with other parts; typical test methods are: visibility, solid sweeping, stereographical projections of c-space obstacles, graph method, electrical field, using floorgraphs, and ray testing. Assembly plan evaluation depends on: tool changes, orientation changes, complexity of the assembly, assembly time, similar assembly operations, cost, and parallelism.

Table 1. Methods for representing assembly plans.

\begin{tabular}{|c|c|l|}
\hline Category & $\begin{array}{c}\text { Type of } \\
\text { representation }\end{array}$ & Representation name \\
\hline \multirow{2}{*}{$\begin{array}{c}\text { One } \\
\text { assembly } \\
\text { sequence }\end{array}$} & Graphical & $\begin{array}{l}\text { Simple indirect graph of } \\
\text { connections }\end{array}$ \\
\cline { 2 - 3 } & Diagram & Assembly trees \\
\cline { 2 - 3 } & List & $\begin{array}{l}\text { List of task } \\
\text { representation }\end{array}$ \\
\cline { 2 - 3 } & List & $\begin{array}{l}\text { List of partitions of the } \\
\text { set of parts }\end{array}$ \\
\cline { 2 - 3 } & List of subset of \\
& connections \\
\hline
\end{tabular}




\begin{tabular}{|c|c|l|}
\hline \multirow{4}{*}{$\begin{array}{c}\text { A set of } \\
\text { assemblies } \\
\text { sequences }\end{array}$} & List & List of binary vectors \\
\cline { 2 - 3 } & Graphical & Directed graphs \\
\cline { 2 - 3 } & Graphical & AND/OR graphs \\
\cline { 2 - 3 } & Dector graph \\
\cline { 2 - 3 } & Diagram & Precedence graphs \\
\cline { 2 - 3 } & Function & $\begin{array}{l}\text { Establishment } \\
\text { conditions }\end{array}$ \\
\cline { 2 - 3 } & List & Precedence relationship \\
\cline { 2 - 3 } & &
\end{tabular}

Table 2. Methods for the generation of assembly plans.

\begin{tabular}{|c|c|}
\hline Category & Description \\
\hline $\begin{array}{l}\text { Precedence } \\
\text { knowledge }\end{array}$ & $\begin{array}{l}\text { Use of precedence constraints to } \\
\text { generate feasible assembly } \\
\text { sequences. }\end{array}$ \\
\hline $\begin{array}{l}\text { Grouping } \\
\text { components }\end{array}$ & $\begin{array}{l}\text { Grouping the components of a } \\
\text { product according to their features } \\
\text { and similarities. }\end{array}$ \\
\hline $\begin{array}{c}\text { Forming } \\
\text { subassemblies }\end{array}$ & $\begin{array}{l}\text { By forming subassemblies based } \\
\text { on some specific preferences. }\end{array}$ \\
\hline $\begin{array}{l}\text { Graphical } \\
\text { approach }\end{array}$ & $\begin{array}{l}\text { Using tracking or cut-set methods } \\
\text { based on graphical representation } \\
\text { of assembly sequence(s) or part- } \\
\text { connection diagram(s). }\end{array}$ \\
\hline Genetic search & $\begin{array}{l}\text { Use of genetic algorithms to obtain } \\
\text { assembly plans. }\end{array}$ \\
\hline $\begin{array}{l}\text { Assembly state } \\
\text { codification }\end{array}$ & $\begin{array}{l}\text { Assembly states are codified and } \\
\text { the assembly sequences are } \\
\text { generated according to this } \\
\text { codification. }\end{array}$ \\
\hline Virtual & Virtual assembly of components. \\
\hline Motion based & $\begin{array}{l}\text { Assembly planning based on a } \\
\text { motion planning method. }\end{array}$ \\
\hline $\begin{array}{l}\text { Random } \\
\text { approach }\end{array}$ & $\begin{array}{l}\text { Use of randomised methods like } \\
\text { the Road Map approach. }\end{array}$ \\
\hline $\begin{array}{c}\text { Feasibility } \\
\text { decompositions }\end{array}$ & $\begin{array}{l}\text { Test the feasibility of separating } \\
\text { the components from the assembly } \\
\text { product. }\end{array}$ \\
\hline
\end{tabular}

The assembly planning systems reported in the literature consist basically of four generic operations: 1) 
assembly sequence generation, 2) assembly sequence evaluation, 3) simulation, and 4) robot instruction generation. The majority of these assembly planners have been tested with a relative small number of components (10 to 20 components).

\subsection{Manual assembly planning}

Historically assembly plans were selected by engineers only after the product design had been completed, approved and authorized; there were some guidelines to plan the manual assembly. Nowadays these assembly guidelines comprise a multidisciplinary combination of experiential, analytical and theory-based recommendations. Human factors are decisive in assembly rationalization. Worker performance is limited in terms of speed, stamina, and accuracy. Manual assembly has been improved by better design of the workplace and substitution of muscle power by other energy sources.

Manual assembly has two advantages: 1) it applies simply and less costly hand tools, and 2) a greater variation in part dimensions can be tolerated. In manual assembly, control of motion, decision-making capability and flexibility, assuming well-trained operators, are superior to current machines. At times, it is economical to provide operators with assistance (fixtures, gauges, computer displays, assembly plans, etc.) to reduce the assembly time and errors. For these reasons, there are still many jobs that companies prefer to assign to humans, such as in the automotive industry. These jobs are often repetitive, involving visual inspection by a single worker at a single station and require dexterity not available to robotic process grippers. Workstations for manual assembly tend to involve bulk, or flexible, materials with relatively more unstructured requirements. 


\subsection{Octree decomposition}

The OcBlox system (Fig. 1) applies Octree decomposition to CAD models or assemblies to turn them into approximate Octree models, Medellín et. al. (2006). The Octree decomposition technique is a hierarchical tessellation that subdivides a volume into octants (cubes) of varying sizes. The relationship among cubes is a hierarchical tree structure, where each branch is identified by the relative position of the octant in its parent node. Octants can be classified as full, empty or boundary, depending on their relative location in the $\mathrm{CAD}$ model: inside, outside or partially inside, respectively. For maximum approximation, full and boundary octants are included in the approximate model. The octree decomposition can be controlled by different criteria such as the maximum level of decomposition or the minimum size of octant. At the end of the decomposition process, the list of octants will form an approximate representation of a CAD model.

In the OcBlox system the octant sizes are limited to particular sizes according to the capability of the manufacturing process. To control the sizes of cubes between a minimum value $s_{\min }$ and a maximum value $s_{\max }$, the OcBlox system uses the fixed-size-range decomposition approach, Medellín et. al. (2006). An optimization process is also performed by the system to reduce the number of cubes in the Octree model. In this optimis ation process each group of eight adjacent cubes is replaced by one larger cube, Medellín et. al. (2008).

\section{Assembly cell}

An assembly cell to build cube-based models was developed as shown in Fig. 2; comprising: 
A. Robotic system: an Epson® SCARA ES653S industrial robot (nominal repeatability $\pm 0.02 \mathrm{~mm}$ ) and an Epson ${ }^{\circledR}$ SRC320 ABS multi-task controller with $16 \mathrm{I} / \mathrm{O}$ that can be read and generated via the SPEL API.

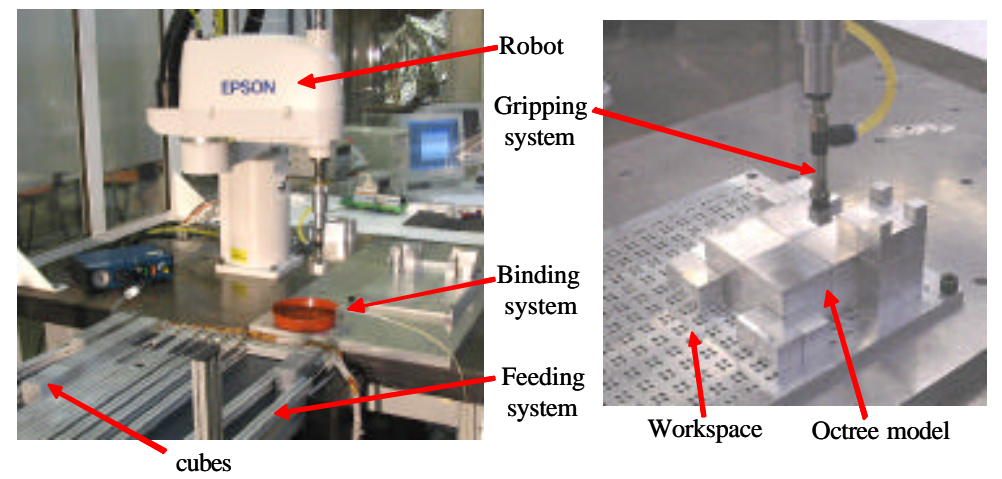

Figure 2. Octree assembly cell.

B. Feeding system: stores and feeds the cubes to the assembly cell using a belt conveyor and several adjustable-width lanes enabling the automatic alignment and placing of the cubes for picking. At each lane a sensor controls the automatic feeding of cubes.

C. Binding system: consisting of an adhesive bath into which each cube being assembled is immersed before its assembly. A cyanoacrylate-based adhesive is used because of its fast curing and high bond strength; in this case Loctite ${ }^{\circledR} 426$ with bond strength to aluminium of 2045 psi and a curing speed of 5 seconds for $40 \%$ of the full cured strength and $60 \%$ of relative humidity (The Design Guide for Bonding Metals, 2004).

D. Gripping system: consists of a vacuum cup (10 mm diameter) attached to the robot arm. This gripper uses the top face of a cube to grasp it but without obstructing other faces so they are clear for glue application and assembly. 
E. Workspace: the current configuration of the assembly cell has a workspace area of $600 \times 300 \times 200$ $\mathrm{mm}$. A square support is fitted at the origin of the workspace to provide alignment during assembly.

F. Raw material: aluminium solid cubes of 10,20 , and $40 \mathrm{~mm}$ are used. These sizes were selected based on the series of preferred numbers ISO R10, the commercial sizes of aluminium square bars, and the dimension of the gripper.

\section{OcBlox Assembly Planning (OAP) system}

The OcBlox Assembly Planning (OAP) system comprises three main modules: a) assembly sequence generator (ASG); b) assembly sequence evaluator (ASE); and c) assembly sequence translator (AST), as shown in Fig. 3. The input data to the OAP system is an octree model which can be obtained by an octree decomposition of the CAD model to be constructed. The output data of the OAP system are the assembly instructions for robot and/or manual assembly.

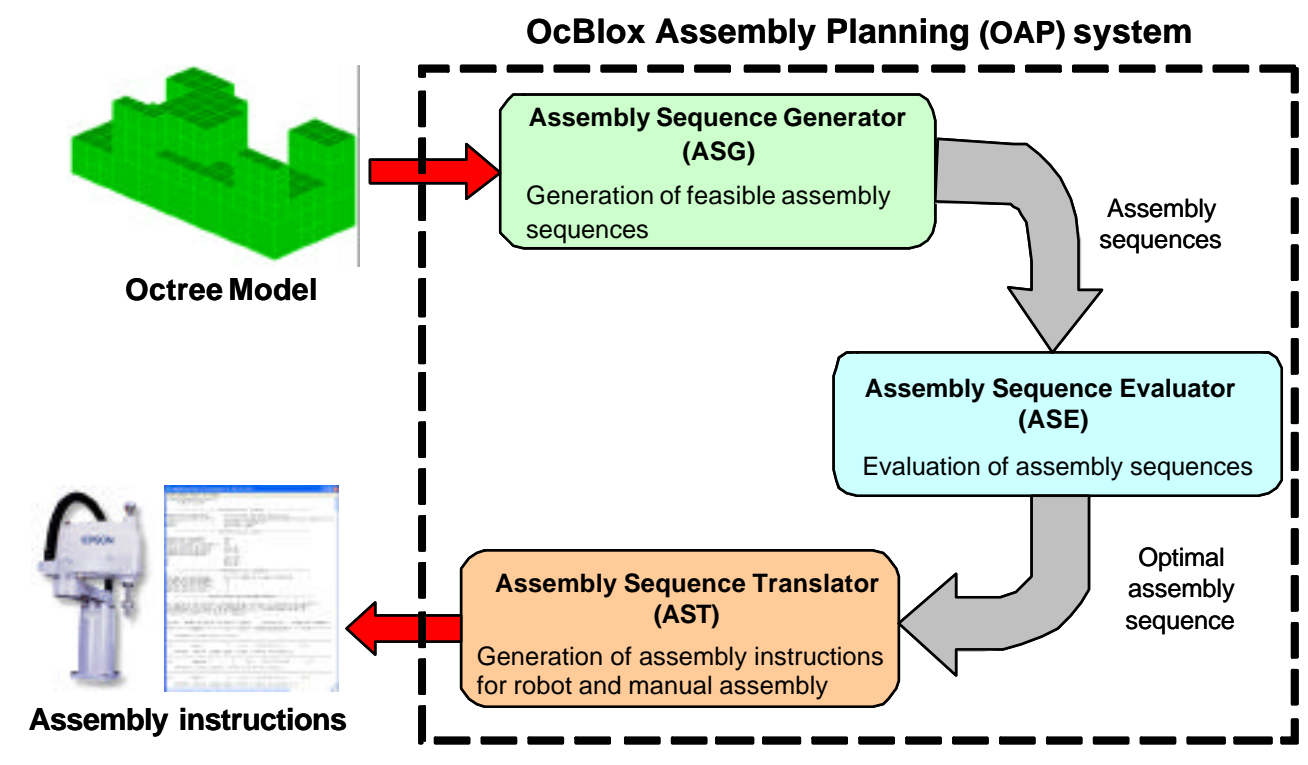


Figure 3. OcBlox assembly planning system.

According to the configuration of the assembly cell (Section 3), a general assembly process was defined by three main moves: $\mathrm{m} 1$ from the feeder to the binding system, $\mathrm{m} 2$ from the binding system to the workspace boundary, and $\mathrm{m} 3$ from the workspace boundary to the final assembly location. Assembly preferences are defined in terms of four assembly variables limited to directions parallel to the coordinate system axes $(+\mathrm{X},+\mathrm{Y},+\mathrm{Z},-\mathrm{X},-\mathrm{Y}$ and $-\mathrm{Z})$, namely:

(i) First axis: progress assembly direction of cubes.

(ii) Second axis: progress direction of the first axis.

(iii) Build axis: progress direction of second axis.

(iv) Assembly trajectory: approach direction to assemble a cube.

The first, second and build axes preferences are used to define the way in which the assembly of a component must progress, and they are used for both robot and manual assembly. On the other hand, the assembly trajectory is just used for the robot assembly since in the manual assembly the person may not follow this preference of the motion.

\subsection{Assembly sequence generator (ASG)}

If octants are always added to the assembly sequence in a predefined collision-free order, the assembly plan will then be geometrically feasible; this is the basis of the modular assembly algorithm, Medellín et. al. (2006), which is used by the ASG to generate feasible assembly sequences of Octree models. An ordered depth first search of an Octree structure allows the generation of an assembly sequence 
using a predefined collision free order of each octant and its children. This octants-assembly order is defined by the assembly variables.

To overcome the problems related to mechanical feasibility, manipulability and accessibility, the following two conditions were established in the assembly sequence generation process: (1) a bottom up build process is always used; (2) no cubes above the height level of the cube being assembled and along the gripper approach direction can be assembled prior to it. These two conditions and the configuration of the assembly cell mean that the possible directions of the assembly growth and the trajectory used to position individual blocks were restricted to 36 discrete combinations. The possible values of the assembly variables are shown in Table 3 , which lead to 36 feasible assembly sequences.

Table 3. Feasible values for the assembly variables.

\begin{tabular}{|c|c|c|c|c|c|c|}
\hline $\begin{array}{c}\text { Assembly } \\
\text { sequences }\end{array}$ & $\begin{array}{c}\text { First } \\
\text { axis }\end{array}$ & $\begin{array}{c}\text { Second } \\
\text { axis }\end{array}$ & $\begin{array}{c}\text { Build } \\
\text { axis }\end{array}$ & \multicolumn{3}{|c|}{$\begin{array}{c}\text { Assembly } \\
\text { trajectory }\end{array}$} \\
\hline $1-3$ & $\mathrm{X}$ & $\mathrm{Y}$ & $\mathrm{Z}$ & $-\mathrm{X}$ & $-\mathrm{Y}$ & $-\mathrm{Z}$ \\
\hline $4-6$ & $\mathrm{X}$ & $\mathrm{Z}$ & $\mathrm{Y}$ & $-\mathrm{X}$ & $-\mathrm{Y}$ & $-\mathrm{Z}$ \\
\hline $7-9$ & $\mathrm{Y}$ & $\mathrm{X}$ & $\mathrm{Z}$ & $-\mathrm{X}$ & $-\mathrm{Y}$ & $-\mathrm{Z}$ \\
\hline $10-12$ & $\mathrm{Y}$ & $\mathrm{Z}$ & $\mathrm{X}$ & $-\mathrm{X}$ & $-\mathrm{Y}$ & $-\mathrm{Z}$ \\
\hline $13-15$ & $\mathrm{Z}$ & $\mathrm{X}$ & $\mathrm{Y}$ & $-\mathrm{X}$ & $-\mathrm{Y}$ & $-\mathrm{Z}$ \\
\hline $16-18$ & $\mathrm{Z}$ & $\mathrm{Y}$ & $\mathrm{X}$ & $-\mathrm{X}$ & $-\mathrm{Y}$ & $-\mathrm{Z}$ \\
\hline $19-21$ & $\mathrm{X}$ & $-\mathrm{Y}$ & $\mathrm{Z}$ & $-\mathrm{X}$ & $\mathrm{Y}$ & $-\mathrm{Z}$ \\
\hline $22-24$ & $\mathrm{X}$ & $\mathrm{Z}$ & $-\mathrm{Y}$ & $-\mathrm{X}$ & $\mathrm{Y}$ & $-\mathrm{Z}$ \\
\hline $25-27$ & $\mathrm{Z}$ & $\mathrm{X}$ & $-\mathrm{Y}$ & $-\mathrm{X}$ & $\mathrm{Y}$ & $-\mathrm{Z}$ \\
\hline $27-30$ & $\mathrm{Z}$ & $-\mathrm{Y}$ & $\mathrm{X}$ & $-\mathrm{X}$ & $\mathrm{Y}$ & $-\mathrm{Z}$ \\
\hline $31-34$ & $-\mathrm{Y}$ & $\mathrm{X}$ & $\mathrm{Z}$ & $-\mathrm{X}$ & $\mathrm{Y}$ & $-\mathrm{Z}$ \\
\hline $34-36$ & $-\mathrm{Y}$ & $\mathrm{Z}$ & $\mathrm{X}$ & $-\mathrm{X}$ & $\mathrm{Y}$ & $-\mathrm{Z}$ \\
\hline
\end{tabular}

The cube fastening mechanism considered is an instant adhesive; therefore, unstable assembly conditions (e.g. overhanging or re-entrant shapes) may cause binding problems affecting the component accuracy 
and rigidity. A stability algorithm was developed to find unstable assembly conditions by considering gravitational forces. When an octant is found to be unstable, the algorithm searches for adjacent empty octants and adds them to the octree model as non-glued supporting cubes that are easily removed after building. The stability algorithm also performs stability checks of supporting cubes being added to avoid new unstable conditions. Adjacent octants are found using the neighbour finding method proposed in Samet Hanan (1989). A part orientation analysis was also developed to analyse different orientations of the octree model and minimise the number of supporting cubes needed. Six orthogonal orientations parallel to the coordinate axes were considered with the optimal orientation being the one requiring the least supports. More details of the stability and part orientation algorithms can be found in Medellín et. al. (2008).

\subsection{Assembly sequence evaluator (ASE)}

The ASE module finds the optimal assembly sequence from the set of feasible assembly sequences previously generated in the ASG module. The assembly sequence evaluation depends on weighted criteria such as: tool changes, orientation changes, fixture complexity, directionality, travelled distance, assembly time, similar assembly operations, cost, and parallelism. Since in an octree model all the parts are cubes assembled using the same tooling, the most significant criterion considered is the travel distance, i.e. the robot's assembly path length, which can be estimated as:

$$
d_{T}=\sum_{i=1}^{n}\left(d_{1 i}+d_{2 i}+d_{3 i}\right)
$$

where $d_{T}$ is the total travel distance, $n$ is the number of cubes to be assembled and $d_{1}, d_{2}$, and $d_{3}$ are the 
travel distances for the motions $\mathrm{m} 1, \mathrm{~m} 2$ and $\mathrm{m} 3$ respectively. These distances depend on the locations of the feeder, the binding system and the assembly trajectory. Since the location of the feeder and the binding system are fixed and depend on the assembly cell configuration, the travel distance will vary only if the assembly trajectory varies. Thus, the ASE module computes the travel distance for each assembly sequence and selects the one with the minimum travelled distance. For manual assembly, this evaluation process can be also used; however, as it was mentioned before, there is no guarantee that the person will perform the assembly in the trajectory defined since humans move intuitively.

\subsection{Assembly sequence translator (AST)}

The AST is responsible for generating the assembly plan or assembly instructions to perform the robot (automatic) and/or manual assembly.

\subsubsection{Automatic assembly}

The AST module generates the robot instructions in the SPEL language of the robot controller which are used in the OAP application program. In this way, the OAP program generates the robot instructions required to perform the assembly, including the control of all the variables required and defined by the user (e.g. speed, acceleration, gripping, glue application, etc.). The synchronization between the robot and the OAP system is direct and there is no need to export or import robot instruction files.

\subsubsection{Manual assembly}

Manual assembly planning considers the generation of the instructions required to manually assemble the octree component. Based on the CAD information of the octree model, the AST module generates the 
manual assembly instructions as a separate text file which can be opened or printed by the user to be used during the assembly process. The assembly instructions file provides the job information: file name, file location, current date and time; the model information: number of parts and supports, total number of parts to be assembled, minimum and maximum size of the parts and overall dimensions of the component; the assembly information coordinate system and assembly preferences; and assembly instructions: assembly operation, part number, size, location, adjacent parts, estimated assembly time, and assembly directions. The assembly operations and parts are ordered and numbered according to the assembly sequence. The assembly instructions comprise information related to the adjacent parts to the part being assembled, information regarding if the part is acting as a support or not, and assembly instructions such as align, place, glue, etc. Figure 4 shows an example of the assembly instructions generated by the system, where the estimated assembly time is also included.

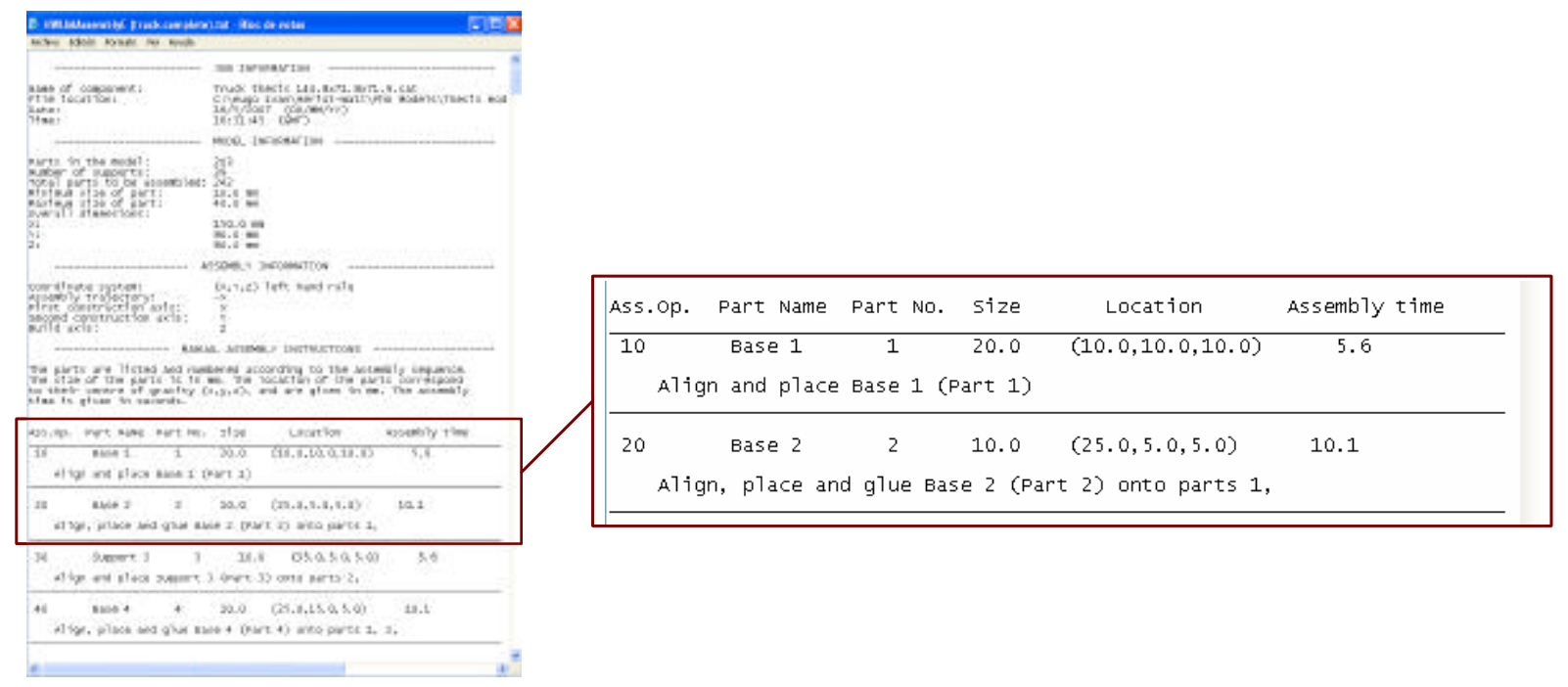

Figure 4. Manual assembly directions generated by the OAP system. 
The assembly time for each manual assembly operation is estimated based on the Methods-Time Measurement (MTM-1) system, ), Niebel and Freivalds (2004), which uses the TMU as the standard unit time $(1 \mathrm{TMU}=0.00001$ hour. The assembly time of an ordinary part and an ordinary support are 280.9 TMU (10.1124 s) and 154.3 TMU (5.5548 s), respectively. These unit times have been estimated based on the configuration of the assembly workspace and the MTM analyses shown in Tables 4 and 5. Based on these analyses, the assembly time required to manually construct a component is estimated by the system.

Table 4. MTM analysis to assemble an ordinary part.

\begin{tabular}{|c|l|c|c|} 
No. & \multicolumn{1}{|c|}{ Description } & $\begin{array}{c}\text { Basic } \\
\text { motion }\end{array}$ & $\begin{array}{c}\text { Time } \\
\text { (TMU) }\end{array}$ \\
\hline 1 & Reach part from feeder & R25B & 22.9 \\
\hline 2 & Get part from feeder & G1B & 3.5 \\
\hline 3 & Move part to the glue system & M12C & 15.2 \\
\hline 4 & Turn part to apply glue on face 1 & M1B & 2.9 \\
\hline 5 & Foot motion to apply glue & FM & 8.5 \\
\hline 6 & Waiting time to apply glue & W & 27.7 \\
\hline 7 & Turn part to apply glue on face 2 & M1B & 2.9 \\
\hline 8 & Foot motion to apply glue & FM & 8.5 \\
\hline 9 & Waiting time to apply glue & W & 27.7 \\
\hline 10 & Regrasp part & G2 & 5.6 \\
\hline 11 & Turn part to apply glue on face 3 & M1B & 2.9 \\
\hline 12 & Foot motion to apply glue & FM & 8.5 \\
\hline 13 & Waiting time to apply glue & W & 27.7 \\
\hline 14 & $\begin{array}{l}\text { Move part to the assembly } \\
\text { workspace }\end{array}$ & M15B & 15.8 \\
\hline 15 & Eye focus on the assembly & EF & 7.3 \\
\hline 16 & Position part & P3NSE & 47.8 \\
\hline 17 & Regrasp part & G2 & 5.6 \\
\hline 18 & Apply pressure & APA & 10.6 \\
\hline 19 & Release part into position & RL1 & 2.0 \\
\hline 20 & $\begin{array}{l}\text { Eye focus on the assembly } \\
\text { instructions }\end{array}$ & EF & 7.3 \\
\hline 21 & Eyes travel to feeder & ET25/15 & 20 \\
\hline & \multicolumn{1}{|c|}{ Total } & 280.9 \\
\hline
\end{tabular}


Table 5. MTM analysis to assemble an ordinary support.

\begin{tabular}{c|l|cc|} 
No. & \multicolumn{1}{|c|}{ Description } & $\begin{array}{c}\text { Basic } \\
\text { motion }\end{array}$ & $\begin{array}{c}\text { Time } \\
\text { (TMU) }\end{array}$ \\
\hline 1 & Reach support from feeder & R25B & 22.9 \\
\hline 2 & Get support from feeder & G1B & 3.5 \\
\hline 3 & $\begin{array}{l}\text { Move support to assembly } \\
\text { workspace }\end{array}$ & M25C & 27.3 \\
\hline 4 & Eye focus on the assembly & EF & 7.3 \\
\hline 5 & Position support & P3NSE & 47.8 \\
\hline 6 & Regrasp support & G2 & 5.6 \\
\hline 7 & Apply pressure & APA & 10.6 \\
\hline 8 & Release support into position & RL1 & 2.0 \\
\hline 9 & $\begin{array}{l}\text { Eye focus on the assembly } \\
\text { instructions }\end{array}$ & EF \\
\hline 10 & Eyes travel to feeder & ET25/15 \\
\hline & \multicolumn{2}{|c|}{ Total } \\
\hline
\end{tabular}

The manual assembly function has been designed for components that have been turned into octrees, but its use can be extended to components that do not have any particular, or regular, shape. The manual assembly function is also able to generate assembly instructions in different languages such as English and Spanish.

\section{Discussion of results}

The OAP system has been implemented in Visual C++ using the ACIS® geometric modelling kernel to support the geometric operations. The User Interface (UI) facilitates interaction and visualization of octree decomposition and optimization, automatic and manual assembly planning, process authoring and testing, assembly instruction generation for robot and manual (English or Spanish) assembly, and performance analysis of the system.

\subsection{Automatic Assembly Planning}


The capability of the OAP system has been tested with the construction of several components. As an example, the construction of the truck component shown in Fig. 5a is presented. The octree decomposition led to an octree model comprising 476 cubes of 10, 20, and $40 \mathrm{~mm}$, Fig. 5b. After optimizing this model, the number of octants was reduced to 203 cubes as shown in Fig. 5c. The stability analysis of this model led to the addition of 39 supports, Fig. 5d. The assembly planning was then carried out and the results suggested an optimal assembly sequence with a first, second, build and assembly trajectory preferences of $+\mathrm{X},+\mathrm{Y},+\mathrm{Z}$ and $-\mathrm{X}$, respectively, and with a travelled distance of 161,098 mm, Fig. 5e. This sequence was used to construct the final component in the robotic assembly cell, Fig. 5f. The time for the robot to assemble the model was 39 minutes, and the total production time including the assembly planning was $1 \mathrm{hr} 5 \mathrm{~min} 29 \mathrm{~s}$.

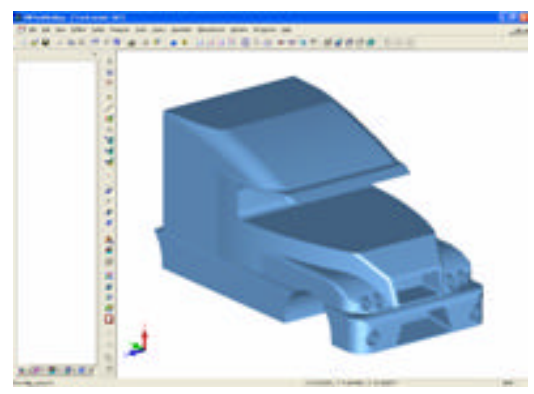

(a)

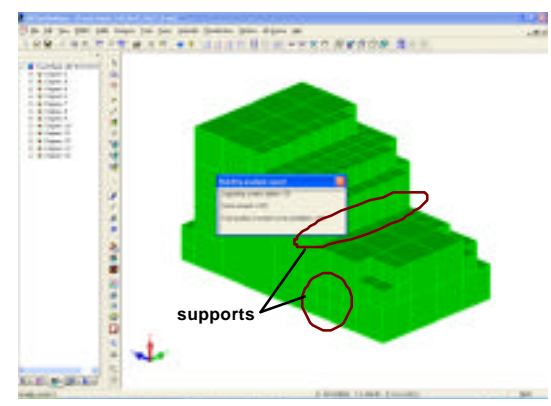

(d)

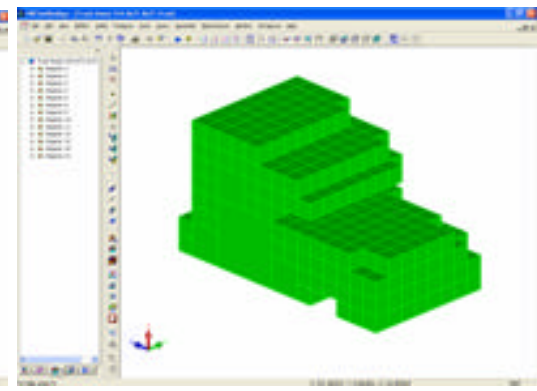

(b)

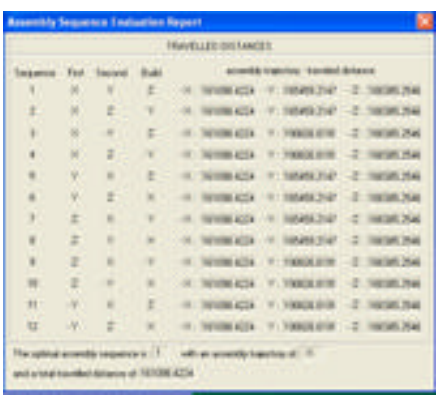

(e)

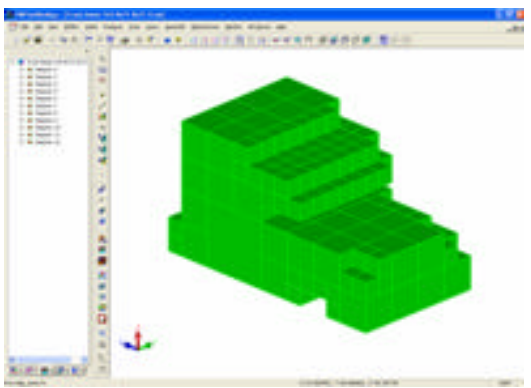

(c)

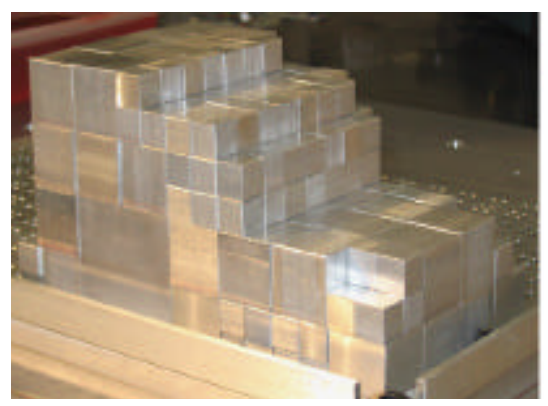

(f)

Figure 5. Truck component: a) CAD model, b) Octree model, c) optimized model, d) addition of 
supports, e) assembly planning, f) final component.

The robot assembly planning has been tested successfully by constructing several components. The results have demonstrated that the OAP system is able to generate feasible assembly plans for a robot to assembly Octree models. The performance of the assembly cell led to an assembly unit time of 9.66 s/cube, which was estimated using the information obtained from the construction of several components.

\subsection{Manual Assembly Planning}

Manual assembly instructions of the truck component were also generated as a separate file as shown in Fig. 6 These instructions detail the assembly operations required to perform the assembly and the information regarding the assembly job. According to this manual assembly plan, 242 manual assembly operations are required involving placing, aligning and gluing the parts at a specific location specified by coordinates and adjacent parts. The assembly time to perform the manual assembly is 2266.6 seconds (37 min $46.6 \mathrm{~s}$ ), which is slightly smaller than the robot assembly time of $39 \mathrm{~min}$. It should be noticed that this assembly time is an estimation based on the assembly units $10.11 \mathrm{~s} / \mathrm{cube}$ and $5.55 \mathrm{~s} / \mathrm{support}$ (MTM analysis of Tables 4 and 5). 


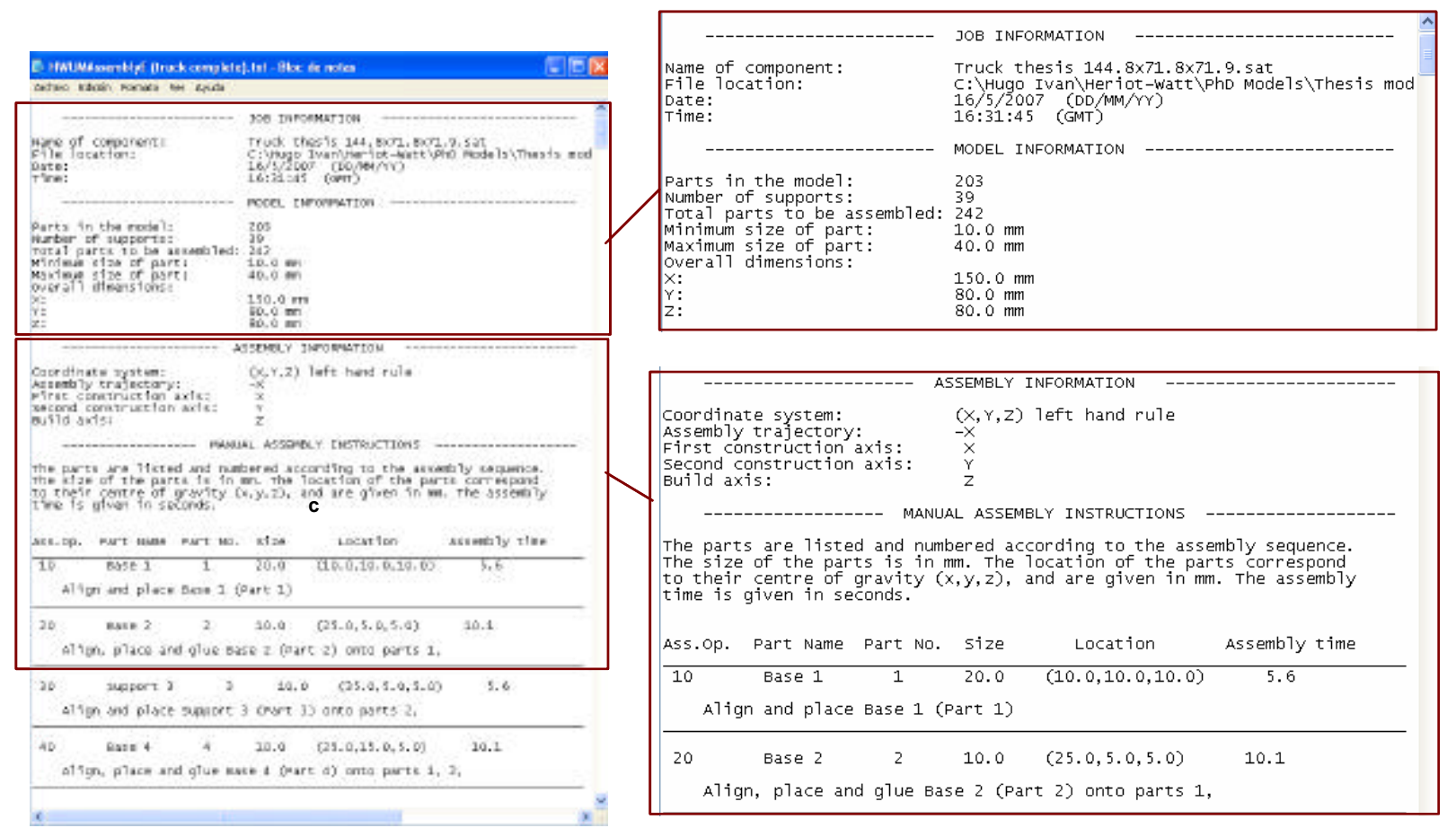

Figure 6. Manual assembly instructions for the truck component.

Several experiments were performed with different people and using a benchmark assembly (which had a smaller number of parts than the truck component). Each person in the experiment performed the assembly three times using just one hand. From the experiments carried out it was observed that the average unit time to assemble a cube was $2.5 \mathrm{~s}$, which is smaller than the estimated time using the MTM analysis (10.11 s) and the time obtained using the robot cell (9.66 s/cube). This difference may be explained as follows. I was observed that the third assembly trial of each person was faster than the first and the second trials, which suggests that people get faster as they get more experience or training. It was also clear that once the people got to know the components to be assembled, they performed the assembly using less motions (i.e. an optimal trajectory) without even having to look at the assembly plan. This ability of human beings to learn and optimise 30 tasks is an important skill that makes them superior 
to automatic machines. Moreover, position, speed and acceleration during the assembly are intuitively calculated by humans and may vary from person to person. On the other hand, robot motions are inflexible so the robot has to perform the assembly using the defined motions even though they may not be the optimal for each part. Another important observation is the difficult and time consuming process of reading assembly instructions when people are performing assemblies with many parts. People suggested including pictures in the assembly plan so they can understand faster the assembly instructions. People do not like reading positions or locations that are provided by coordinates, they prefer pictures instead. Tiredness is an important aspect that should be taken into account when components with large number of parts are assembled by humans. This is an advantage of robots over humans since they are machines and tiredness does not have an impact on their performance. In these experiments tiredness was not evaluated.

\subsection{OAP system performance}

The OAP system has been proved to be a feasible robot and manual assembly planning system. The performance of the system has been evaluated using several components and the results have indicated the following general performance values:

- Time to generate an assembly sequence: $0.843 \mathrm{~s}$.

- $\quad$ Time to evaluate the assembly sequences: $3.78 \mathrm{~s}$.

- Time to translate an assembly sequence: $2 \mathrm{~min}$.

These values were estimated with the construction of several components, having a 289 cubes octree model as an average. From these values it can be said that the system is relatively fast. This is because 
the assembly planning does not make use of complex operations, such as Boolean operations, ray firing, sweeping, genetic algorithms, etc., that are computationally expensive and that are used by most of the assembly planners reported in the literature, see Table 2. Thus, it can be said that the otree decomposition represents a technique that can be used for fast assembly planning of a large number of parts. However, one disadvantage of this technique is that it approximates a part instead of using the actual part. On the other hand, when a component comprising several parts is intended to be assembled, the system should be able to identify the independent parts and decomposed them separately. Then, the octree models of the parts should be assembled in an environment surrounded by these octree models. In other words, the system should be able to plan the assembly of several octree models. For this reason, more research work is being carried out to solve the current limitations of the OAP system.

\section{Conclusion}

The octree decomposition technique has been proved to be an effective assembly planning tool. As a result, an assembly planning system, named as OcBlox Assembly Planning (OAP) system, has been developed and presented in this paper. After the implementation of the OAP system, the results have showed that the assembly planning of CAD models for either robot or manual assembly can be performed using an octree decomposition approach. Assembly plans for automatic and manual assembly can be generated automatically by the proposed system, which is a novelty since there are no assembly planning systems for manual assembly reported in the literature. Moreover, since the OAP system is based on the octree data structure and does not use any computationally expensive algorithm, 
the assembly planning is relatively fast and it can be performed on components comprising hundreds of parts.

Future work will focused in the solution of the current limitations of the OAP system, including the generation of manual assembly plans with pictures, assembly precedence diagrams for cube-based fabrications, e.g. Soma puzzles or CAD assemblies that have been turned into Octrees, Sung (2001). Also, the potential applications to support cube construction at micro or macro scales including the precision of manipulators and joining technologies, is also considered as future work.

\section{Acknowledgment}

This research work was supported by the EPSRC grant GR/R35285/01. The first author acknowledges the support from the Fund for Research Support (FAI) of the Universidad Autónoma de San Luis Potosí and the support from the PROMEP program of SEP in Mexico.

\section{References}

Chen Y. H., Song Y., "The development of a layer based machining system” Computer Aided Design, 33, 2001, pp. 331-342.

Frank Matthew, Sanjay B. Joshi, and Richard A. Wysk, "CNC-RP: A technique for using CNC machining as a rapid prototyping tool in product/process development", Proceedings of the 11th annual industrial engineering research conference, Orlando, Florida. May 19-22, 2002.

Gibson Ian, "A discussion on the concept of a flexible rapid prototyping cell”, Rapid Prototyping Journal, 2 (2), 1996, pp. 32-38. 
Homem de Mello Luiz S., Lee Sukhan, “Computer-Aided Mechanical Assembly Planning”, foreword by George A. Bekey, Kluwer Academic Publishers, 1991.

Hsuan-kuan Huang, Lin Grier C. I., "Rapid and flexible rapid prototyping through a dual robot workcell", Robotics and Computer Integrated Manufacturing, 19, 2003, pp. 263-272.

Junghoon Hur, Lee Kunwoo, Zhu-hu, Kim Jongwon, "Hybrid rapid prototyping system using machining and deposition", Computer Aided Design, 34, 2002, pp. 741-754.

Kai-I Huang and Tai-His Wu, "Computer-Aided Process Planning for Robotic Assembly", 17th International Conference on Computers and Industrial Engineering, 29, 1-4, 1995, pp. 653-657. Karunakaran K.P., P. Vivekananda Shanmuganathan, Sanjay Janardhan Jadhav, Prashant Bhadauria, Ashish Pandey, "Rapid prototyping of metallic parts and moulds", Journal of Materials Processing Technology, 105, 2000, 371-381.

Kaufman Stephen G., Wilson Randall H., Jones Rondall E., Calton Terri L and Ames Arlo L., "The Archimedes 2 Mechanical Assembly Planning System", Procc. of the 1996 IEEE International Conference on Robotics and Automation, 1996, pp. 3361-3368.

Lin Y. J. and Farahati R., "CAD-Based Virtual Assembly Prototyping - A Case Study", The International Journal of Advanced Manufacturing Technology, 21, 2003, pp. 263-274.

Mascle Christian, "Feature-based assembly model for integration in computer-aided assembly", Robotics and Computer Integrated Manufacturing, 18, 2002, pp. 373-378.

Medellín H., Corney J., Davies J.B.C., Lim T., Ritchie J. M., “Algorithms for the physical rendering and assembly of octree models", Computer Aided Design, 38, 2006, pp. 69-85. 
Medellín H., Corney J., Davies J.B.C., Lim T., Ritchie J.M., “Octree Based Production of Near Net Shape Components", IEEE Transactions on Automation Science and Engineering, vol. 5, No. 3, July 2008, pp. 457-466.

Niebel B. W., Freivalds A. Ingeniería Industrial, Métodos estándares y. diseño del trabajo. Ed. Alfaomega. ED. 11. a. México 2004

Samet Hanan, "Neighbour finding in images represented by octrees", Computer Vision, Graphics and Image Processing, 46, 1989, pp. 361-386.

Schmidt, L. C., Jackman, J., "Evaluating assembly sequences for automatic assembly systems”, IIE Transactions, 27, 1995, pp. 23-31.

Sundaram Sujay, Remmler Ian and Amato Nancy M., "Disassembly Sequencing Using a Motion Planning Approach", Proceedings of the 2001 IEEE International Conference on Robotics and Automation, May 21-26, 2001, pp. 1475-1480.

Sung, R. C. W., “Automatic Assembly Feature Recognition and Disassembly Sequence Generation”, Ph.D. Thesis, Heriot-Watt University, Edinburgh, Scotland, 2001.

Swaminathan Arun and Suzanne Barber K., "An Experience-Based Assembly Sequence Planner for Mechanical Assemblies", IEEE Transactions on Robotics and Automation, 12, 2, 1996, pp. 252-267.

"The Design Guide for Bonding Metals", Loctite®, Henkel Technologies, 4, 2004, http://www.loctite.com

Whitney D.E., Mantripragada R., Adams J. D. and Rhee S. J., "Designing assemblies", Research in Engineering Design, 11, 1999, pp. 229-253. 
Wilson Randall H., "Geometric Reasoning about Assembly Tools”, Technical report SAND95-2423, Sandia National Laboratories, 1996. 